\title{
On the Harmonic Oscillation of High-order Linear Time Invariant Systems
}

\author{
B.B. Alagoz and H.Z. Alisoy
}

\begin{abstract}
Linear time invariant (LTI) systems are widely used for modeling of dynamics systems in science and engineering problems. Harmonic oscillation of LTI systems is an outstanding case of LTI system behavior and it is employed for modeling of many periodic physical phenomenon in nature. This study investigates sufficient conditions to obtain harmonic oscillation by using high-order LTI systems. A design procedure for controlling harmonic oscillation of single-input single-output high-order LTI systems is presented. LTI system coefficients are calculated by solving equation sets, which imposes a stable sinusoidal oscillation solution for the characteristic polynomials of LTI systems. Moreover, these analyses are extended to fractional order LTI systems. Simulation examples are demonstrated for high-order LTI systems and the control of harmonic oscillations are discussed by using Hilbert transform and spectrogram of oscillation signals.
\end{abstract}

Index Terms - Harmonic oscillation, system theory, fractionalorder LTI systems, system modeling, root locus analysis.

\section{INTRODUCTION}

$\mathrm{L}$ INEAR Time Invariant (LTI) system modeling methods have been played an important role in development of science and technology for a century. LTI models have found a widespread utilization in theoretical and numerical analyses of linear dynamic systems. Because, behaviors of dynamic systems can be well characterized by LTI system models and consistency of LTI system analyses with real systems has been proven for numerous applied science and engineering problems. It is obvious that analyses on the base of LTI systems still play a central role in system science, today. Deepened investigation on behaviors of LTI systems promises further contributions in term of modeling and comprehension of physical and electrical system behaviors.

LTI systems are expressed in the form of linear timeinvariant differential equations. It is convenient to represent LTI differential equations as a set of first-order differential equations to perform state space analyses. Besides, Laplace transform and transfer functions are mainly preferred because of simplification of the system analyses [1-3]. The characteristic polynomial of the LTI systems provides a valuable tool for the analysis of the character of LTI system

Baris Baykant Alagoz, was with Inonu University, Department of ElectricalElectronics, Malatya, Turkey (e-mail: baykant.alagoz@inonu.edu.tr).

Hafiz Z. Alisoy, was with Namı Kemal University, Department of Electronics and Telecomunication, Tekirdag, Turkey (e-mail: alismpei@gmail.com).
[4]. Roots of characteristic polynomial are referred to as eigenvalues (or system poles) of systems. Complex conjugate roots $\left(\lambda_{i}=\sigma_{i} \pm j \omega_{i}\right)$ lead to harmonic terms, $e^{j w t}$, in the solution of LTI models, and this results in harmonic components at system output [4,5]. The short-term oscillations, originated from the terms of $e^{\sigma_{i} t \pm j w t}$ for $\sigma_{i}<0$, is transient and they tend to asymptotically damp down in time. A continuous oscillating component at LTI system solutions appears when $\sigma_{i}=0 \quad[4,5]$. The current study is devoted for the design considerations of high-order LTI harmonic oscillators emerging in the cases of $\sigma_{i}=0$ and $j \omega_{i} \neq 0$.

Previously, stability boundary locus analyses were used for the design of stable control systems [6,7]. It is based on solving characteristic polynomial equation in $\mathrm{s}$ domain for $s=j \omega$ to figure out stability regions of controller coefficients. Indeed, stability boundary locus also indicates to the coefficients that results in oscillation of control systems. We consider stability boundary as the oscillation locus and extend our investigation for the harmonic oscillation of LTI systems.

System oscillation is one of the most prominent concerns for dynamic systems. Oscillation behavior is frequently seen in nature, and oscillation of LTI systems was extensively investigated due to potentials of modeling simplification and analysis consistency. Many applied science and engineering fields have been benefited from oscillation models such as in physics [8-12], in control science [13-16], in electrical system $[16,17]$, in biology [19-21]. For instance, prevalence of harmonic oscillators in physics is obvious, such as while describing motions of an object attached to a string and molecules vibrating in crystals [9]. Oscillation conditions were stated for various oscillators [17-19]. However, investigation of parametric conditions to constitute harmonic oscillation (steady sinusoidal oscillations) of high-order LTI models may further extend our comprehension of complex physical systems, which is, today, modeled by simple second-order harmonic oscillation models, particularly in particle physics [9].

This study investigates sufficient conditions to obtain harmonically oscillating high-order LTI system model. Roots of the characteristic polynomials are mapped to amplitudeangle $(M-\theta)$ plane by considering roots of characteristic polynomial in the form of $\lambda=M e^{j \theta}$, and coefficients of the LTI system let to a desired harmonic oscillation were found by arbitrary solutions of linear equations. These arbitrary 
solutions impose sinusoidal oscillation solutions for characteristic polynomials. These solutions are extended for fractional order LTI systems. Example designs were demonstrated for integer and non-integer order LTI systems, and results were discussed for the control of harmonic oscillations.

\section{Methodology}

\section{A. Theoretical Background}

Differential equations of LTI control systems with constant coefficients $\alpha_{i} \in R$ and a derivative order $i[1,2,5]$ are expressed in a general form as follows,

$$
\sum_{i=0}^{n} \alpha_{i} y^{(i)}=u
$$

A LTI system is commonly expressed via state space representation [4] as follows

$$
\begin{aligned}
& x^{\prime}=A x+B u \\
& y=C x+D u,
\end{aligned}
$$

,where $x$ is $n \times 1$ state variable vector of the system and $y$ is the system output vector. The tem $x^{\prime}$ denotes the first derivatives of state variable vector. The vector $u$ represents input vector of the system. The matrix $A$ is $n \times n$ size state transition matrix of system. The matrix $B, C$ and $D$ are model parameters of the dynamic system. For single-input single-output systems, Laplace transform transfer function $[4,22,23]$ was expressed in s-domain domain as,

$$
T(s)=\frac{Y(s)}{U(s)}=C(s I-A)^{-1} B+D
$$

Characteristic polynomial of this system [4] were expressed as follows,

$$
\Delta(\lambda)=\operatorname{det}(\lambda I-A)
$$

$\lambda_{i} \in C, i=0,1,2,3, . ., n-1$ is complex eigenvalues of dynamic systems. In order to solve $\lambda_{i}$, one expresses characteristic equation in polynomial form as follows,

$$
\Delta(\lambda)=\sum_{i=0}^{n} \alpha_{i} \lambda^{i}=0
$$

The roots of characteristic polynomial yield $\lambda_{i}=\sigma_{i} \pm j \omega_{i}$ complex eigenvalues. For a zero input case $(u(t)=0)$, the time domain solution of LTI system contains sum of $e^{\lambda_{i} t}$ terms, $y(t)=a_{0} e^{\lambda_{0} t}+a_{1} e^{\lambda_{1} t}+. .+a_{n-1} e^{\lambda_{n-1} t}$ [4]. Accordingly, the term $e^{\lambda_{i} t}$ can be factored as two components as $e^{\sigma_{i} t} e^{j \omega_{i} t}$. Here, the terms $e^{j \omega_{i} t}$ generates harmonics at the output because of $e^{ \pm j \omega_{i} t}=\cos \left(\omega_{i} t\right) \pm j \sin \left(\omega_{i} t\right)$ and this results in sinusoidal components at the angular frequency of $\omega_{i}=2 \pi f_{i}$. The component $e^{\sigma_{i} t}$ specifies evolution of amplitude of $e^{j \omega_{i} t}$ in time. In the case of $\sigma_{i}<0$, it is also commonly referred to as attenuation coefficient and correspondingly time constant of asymptotic attenuation was commonly considered as $\tau_{i}=-1 / \sigma_{i}$ for physical and electrical system models. These systems damp down any excitation in a limited time period. When $\sigma_{i}>0$, the term of $e^{\sigma_{i} t}$ exponentially grows the amplitude of harmonics $e^{j \omega_{i} t}$ in time and indicates to instability of the system. If $\sigma_{i}=0$, it stabilizes amplitude of the harmonics with the frequency of $\omega_{i}$. It is well known that root locations in complex plane $(\sigma, j \omega)$ tells us following remarks for high-order system behaviors $[1,4,5]$,

(i) If all roots accommodates on the left half plane, so $\operatorname{Re}\left\{\lambda_{i}\right\}=\sigma_{i}<0, \quad i=0,1,2,3, n-1$, the system will be asymptotic stability [4]. Because, all $e^{\sigma_{i} t}$ terms in solution go to zero, as the time goes infinity $\left(\lim _{t \rightarrow \infty} e^{\sigma_{i} t} \rightarrow 0\right)$ [4]. If there is at least a root, of which $\omega_{i} \neq 0$, it yields a transient oscillation with time constant $\tau=-1 / \sigma_{i}$.

(ii) If there is at least a root accommodating on the right half plane, so $\operatorname{Re}\left\{\lambda_{k}\right\}=\sigma_{k}>0, k \in[0, n-1]$, the system will be unstable. Because, at least a term $e^{\sigma_{k} t}$ in solution goes infinitive as time goes infinity. If $\omega_{k} \neq 0$, it yields a unstable oscillation with a growing amplitude.

(iii) If there are not a complex root at right half plane and at least a complex conjugate root accommodates on complex axis $\left(j \omega_{i}\right) ; \operatorname{Re}\left\{\lambda_{i}\right\}=0$ and for the rest of roots satisfies $\operatorname{Re}\left\{\lambda_{k}\right\}=\sigma_{k}<0, \quad k \neq i \quad$ and $k \in[0, n-1], \quad$ this system oscillates. Because, the solution contains at least one harmonic term $\left(e^{j w t}\right)$, which leads to sinusoidal oscillations in time domain solutions.

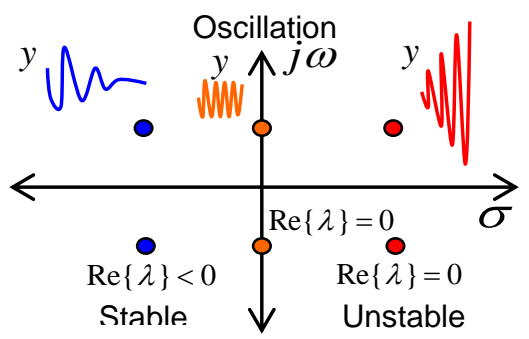

Fig. 1. System responses with respect to root location of characteristic polynomial

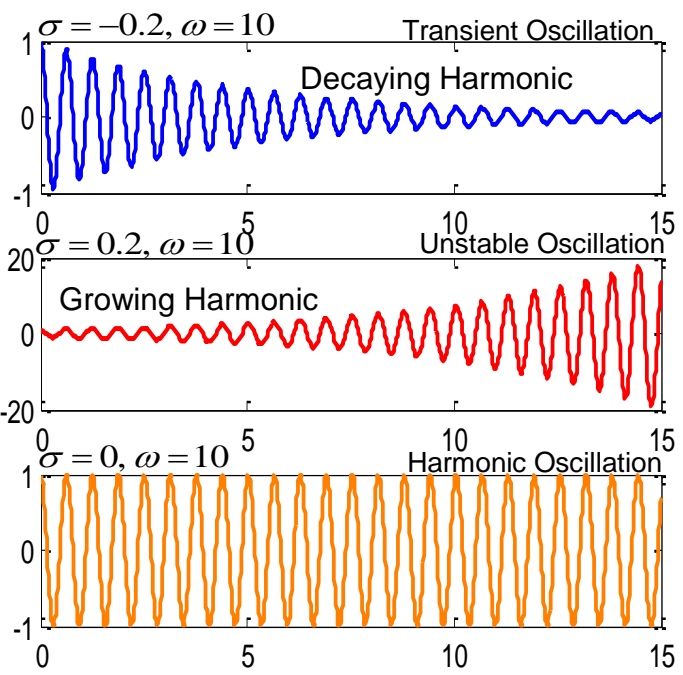

Fig. 2. Oscillation of LTI system for various states of complex roots 
$(\lambda=\sigma \pm j \omega)$

The system responses are represented with respect to root location of characteristic polynomials in Figure 1. Figure 2 illustrates simulation results obtained for sinusoidal oscillation stereotypes with respect to complex roots.

\section{B. Problem Definition}

This study aims to derive fundamental design considerations for harmonic oscillation of high-order LTI systems on the bases of characteristic root placement in complex plane. For this propose, lets express the roots of characteristic polynomial in the form of $\lambda_{i}=M_{i} e^{j \theta_{i}}[24,25]$. Here, $M_{i}$ and $\theta_{i}$ are magnitude and angle of complex roots $\lambda_{i}$, respectively. Transformation from $(M, \theta)$ to $(\sigma, j \omega)$ is performed by using,

$$
\left[\begin{array}{l}
\sigma \\
\omega
\end{array}\right]=M\left[\begin{array}{l}
\cos \theta \\
\sin \theta
\end{array}\right]
$$

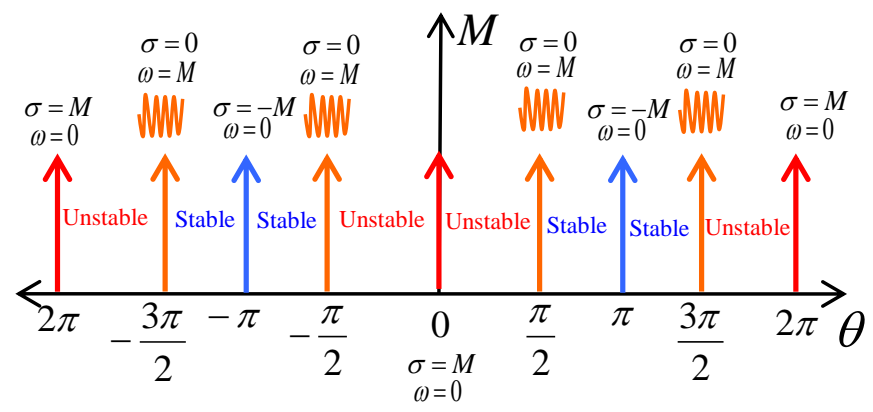

Fig. 3. Root mapping in $M-\theta$ plane and resulting oscillations boundaries

Figure 3 illustrates the character of systems in $M-\theta$ root mapping regions. According to figure, regions of $M>0$ and $\frac{\pi}{2}+h 2 \pi<\theta_{i}<\frac{3 \pi}{2}+h 2 \pi$ for $h \in T$ yields a asymptotically stable LTI systems due to coinciding to left half of complex root plane and regions of $M>0$ and $-\frac{\pi}{2}+h 2 \pi<\theta_{i}<\frac{\pi}{2}+h 2 \pi$ for $h \in T$ yield unstable LTI systems because of mapping right half of complex root plane. Boundaries between stability and instability mapping regions are referred to harmonic oscillating boundaries due to $\omega_{i}=M$ and $\sigma_{i}=0$. Oscillation-free response of LTI system is possible when all $\lambda_{i}$ is mapped over the boundaries of $\theta_{i}=\pi \pm h 2 \pi, i=0,1,2,3, n-1$ due to $\omega_{i}=0$ and $\sigma_{i}=-M$.

Real physical systems in macro scales (mechanical systems) mainly exhibit attenuating harmonic oscillation $\left(\sigma_{i}<0\right)$ for a limited time interval due to friction forces or thermodynamic energy loses. Time constant of this limited time oscillation (transient oscillation) is $\tau=-1 / \sigma_{i}=-1 /\left(M_{i} \cos \left(\theta_{i}\right)\right)$ and the oscillating frequency is $\omega_{i}=M_{i} \sin \left(\theta_{i}\right)$. Steady oscillations can be frequently possible in micro-scale physical systems such as electro-physical and particle systems. However, unstable harmonic oscillation ends with devastation or transformation of the physical systems and this type behaviors can be take place in very short intervals in the nature for instance short-lived particles.

\section{Oscillation Locus of LTI systems}

Property 1: A LTI system yields steady oscillation if at least one root angle satisfies $\theta_{i}=\frac{\pi}{2}+h \pi, i=0,1,2,3,, n-1$ and $h \in T$.

Proof: One can write the angle $\lambda_{i}$ as $\theta_{i}=\tan ^{-1}\left(\omega_{i} / \sigma_{i}\right)$ and use it in $\theta_{i}=\frac{\pi}{2}+h \pi$. In this case, $\sigma_{i}$ can be written as,

$$
\sigma_{i}=\omega_{i} / \tan \left(\frac{\pi}{2}+h \pi\right)
$$

Since $\tan \left(\frac{\pi}{2}+h \pi\right)=\infty$ for $h \in T$, one obtains $\sigma_{i}=0$ for

$h \in T$. Considering remark (iii), this LTI system oscillates due to $\lambda_{i}= \pm j \omega$.

Property 2: A n-order LTI system contains harmonic oscillation at the angular frequency $\omega_{k}$ for the first oscillation locus ( $h=0$ ), when the characteristic polynomial coefficients $\alpha_{0}, \alpha_{1}, \alpha_{2} . ., \alpha_{n}$ satisfy the following conditions,

$$
\sum_{i=0}^{n} \alpha_{i}\left(\omega_{k}\right)^{i} \cos \left(\frac{\pi}{2} i\right)=0 \text { and } \sum_{i=0}^{n} \alpha_{i}\left(\omega_{k}\right)^{i} \sin \left(\frac{\pi}{2} i\right)=0
$$

Proof:

If the characteristic equation of $\mathrm{n}$-order LTI system given by equation (6) is solved for $\lambda_{i}=M_{i} e^{j \theta_{i}}$ mapping, we obtains,

$$
\Delta(\lambda)=\sum_{i=0}^{n} \alpha_{i}\left(M_{i} e^{j \theta}\right)^{i}=0
$$

Considering Property 1 for the first oscillation locus of system $(h=0)$, one can write the following equation,

$$
\sum_{i=0}^{n} \alpha_{i}\left(M_{i}\right)^{i} e^{\left(j \frac{\pi}{2}\right) i}=0
$$

Since, $e^{j\left(\frac{\pi}{2}\right) i}=\cos \left(\frac{\pi}{2} i\right)+j \sin \left(\frac{\pi}{2} i\right)$, equation (10) can be reorganized as follows,

$$
\sum_{i=0}^{n} \alpha_{i}\left(M_{i}\right)^{i} \cos \left(\frac{\pi}{2} i\right)+\sum_{i=0}^{n} \alpha_{i}\left(M_{i}\right)^{i} \sin \left(\frac{\pi}{2} i\right)=0
$$

For $\theta_{i}=\frac{\pi}{2}$, we can write the magnitude as desired oscillation frequency, $M_{i}=\omega_{k}$, and rearrange equation (11) as, 


$$
\sum_{i=0}^{n} \alpha_{i}\left(\omega_{k}\right)^{i} \cos \left(\frac{\pi}{2} i\right)+j \sum_{i=0}^{n} \alpha_{i}\left(\omega_{k}\right)^{i} \sin \left(\frac{\pi}{2} i\right)=0
$$

A solution of equation (12) yields the sufficient conditions of harmonic oscillation at the angular frequency $\omega_{k}$ as,

$$
\sum_{i=0}^{n} \alpha_{i}\left(\omega_{k}\right)^{i} \cos \left(\frac{\pi}{2} i\right)=0 \text { and } \sum_{i=0}^{n} \alpha_{i}\left(\omega_{k}\right)^{i} \sin \left(\frac{\pi}{2} i\right)=0
$$

Property 2 gives the sufficient conditions for determination of characteristic polynomial coefficients in order to make any $\mathrm{n}$ order LTI system contain harmonic oscillation at the angular frequency $\omega_{k}$.

One can write equation (13) in open form as,

when the order $\mathrm{n}$ are even,

$$
\begin{aligned}
& \quad \alpha_{0}-\alpha_{2}\left(\omega_{k}\right)^{2}+\alpha_{4}\left(\omega_{k}\right)^{4}-\alpha_{6}\left(\omega_{k}\right)^{6}+\ldots \\
& \quad \pm \alpha_{n-2}\left(\omega_{k}\right)^{n-2} \pm \alpha_{n}\left(\omega_{k}\right)^{n}=0 \\
& \alpha_{1}-\alpha_{3}\left(\omega_{k}\right)^{3}+\alpha_{5}\left(\omega_{k}\right)^{5}-\alpha_{7}\left(\omega_{k}\right)^{7}+\ldots \\
& \pm \alpha_{n-3}\left(\omega_{k}\right)^{n-3} \pm \alpha_{n-1}\left(\omega_{k}\right)^{n-1}=0
\end{aligned}
$$

When the order $\mathrm{n}$ are odd,

$$
\begin{aligned}
& \alpha_{0}-\alpha_{2}\left(\omega_{k}\right)^{2}+\alpha_{4}\left(\omega_{k}\right)^{4}-\alpha_{6}\left(\omega_{k}\right)^{6}+\ldots \\
& \quad \pm \alpha_{n-2}\left(\omega_{k}\right)^{n-2} \pm \alpha_{n-1}\left(\omega_{k}\right)^{n-1}=0 \\
& \alpha_{1}-\alpha_{3}\left(\omega_{k}\right)^{3}+\alpha_{5}\left(\omega_{k}\right)^{5}-\alpha_{7}\left(\omega_{k}\right)^{7}+\ldots \\
& \pm \alpha_{n-2}\left(\omega_{k}\right)^{n-2} \pm \alpha_{n}\left(\omega_{k}\right)^{n}=0
\end{aligned}
$$

These equations also tell us the following important remarks for oscillation condition of LTI systems:

(i) According to equation (13), due to arbitrary solutions, it is always possible to find out a characteristic polynomial coefficients set $\alpha_{0}, \alpha_{1}, \alpha_{2} . ., \alpha_{n}$ in order to oscillate any LTI system with $n>1$ at any $\omega_{k}$. (Existence of solution)

(ii) According to equation (13), the characteristic polynomial coefficients $\alpha_{0}, \alpha_{1}, \alpha_{2} . ., \alpha_{n}$, which oscillates any LTI system at any $\omega_{k}$, is not unique. So, there is a solution family of $\alpha_{0}, \alpha_{1}, \alpha_{2} . ., \alpha_{n}$ satisfying equation (13). (Abundance of solution)

(iii) A special case of oscillating coefficients of characteristic polynomial appears when $\omega_{k}=1$. A solution family can be obtained as $\alpha_{0}=\alpha_{1}=\alpha_{2}=\ldots=\alpha_{n} \quad$. (Balanced solution families) when the order $\mathrm{n}$ are even,

$$
\begin{array}{r}
\alpha_{0}-\alpha_{2}+\alpha_{4}-\alpha_{6}+\ldots \pm \alpha_{n-2} \pm \alpha_{n}=0 \quad \& \\
\alpha_{1}-\alpha_{3}+\alpha_{5}-\alpha_{7}+\ldots \pm \alpha_{n-3} \pm \alpha_{n-1}=0
\end{array}
$$

when the order $\mathrm{n}$ are odd,

$$
\begin{gathered}
\alpha_{0}-\alpha_{2}+\alpha_{4}-\alpha_{6}+\ldots \pm \alpha_{n-3} \pm \alpha_{n-1}=0 \quad \& \\
\alpha_{1}-\alpha_{3}+\alpha_{5}-\alpha_{7}+\ldots \pm \alpha_{n-2} \pm \alpha_{n}=0
\end{gathered}
$$

(iv) Oscillation condition given by Equation (13) let to arbitrary selection of $n-1$ coefficient and the rest two coefficients are determined by the following equations,

$$
\begin{gathered}
\alpha_{v}=-\sum_{i=0 \& i \neq v}^{n} \alpha_{i}\left(\omega_{k}\right)^{i} \cos \left(\frac{\pi}{2} i\right) \& \\
\alpha_{g}=-\sum_{i=0 \& i \neq g}^{n} \alpha_{i}\left(\omega_{k}\right)^{i} \sin \left(\frac{\pi}{2} i\right)
\end{gathered}
$$

In order to avoid arbitrary locating of $n-2$ roots of characteristic polynomial and negative effects of this root on harmonic oscillation patterns, we prefer to locate them at $\theta_{i}=\pi$ with magnitudes $M_{1}>M_{2}>\ldots>M_{n-2}$, which mean to $\omega=0$ and $\sigma_{1}<\sigma_{2}<\ldots<\sigma_{n-2}<0$. Those roots yield transient asymptotic solutions $\left(e^{\sigma_{i} t}\right)$ approximating to zero without any oscillation as time goes to infinity. In other words, we obtain $n$ number of linear equations for $n$ roots and choose only $\alpha_{0}$ arbitrary. Additional linear equation for each non-oscillating transient roots $(p=1,2 . . n-2)$ can be written as,

$$
\sum_{i=0}^{n} \alpha_{i}\left(\sigma_{p}\right)^{i} \cos (\pi i)=0
$$

This equation is useful for vibration free system design. When all roots of a system satisfies this condition, the system response cleans from transient damping harmonics and rippling.

\section{Extension of Analyses to Fractional Order LTI systems}

Fractional-order system modeling attracts interest of researches, recently. Results in previous section can be easily extended for fractional-order LTI system expressed in a general form as follows

$$
\sum_{i=0}^{n} \alpha_{i} y^{\left(\sigma_{i}\right)}=u
$$

Characteristic equation of fractional-order LTI system is written in the following general form,

$$
\Delta(\lambda)=\sum_{i=0}^{n} \alpha_{i} \lambda^{\sigma_{i}}=0
$$

By considering $\lambda_{i}^{\sigma_{i}}=M_{i}^{\sigma_{i}} e^{j \theta_{i} \sigma_{i}}$, where $\sigma_{i} \in R$ represents fractional orders.

$$
\sum_{i=0}^{n} \alpha_{i}\left(M_{i}^{\sigma_{i}}\right)^{i} e^{\left(j \theta_{i} \sigma_{i}\right)}=0
$$

For $\theta_{i}=\frac{\pi}{2}$, we can write the magnitude as desired oscillation frequency, $M_{i}=\omega_{k}$, and obtains the oscillation condition corresponding to equation (13),

$$
\sum_{i=0}^{n} \alpha_{i}\left(\omega_{k}\right)^{\sigma_{i}} \cos \left(\frac{\pi}{2} \sigma_{i}\right)=0 \text { and } \sum_{i=0}^{n} \alpha_{i}\left(\omega_{k}\right)^{\sigma_{i}} \sin \left(\frac{\pi}{2} \sigma_{i}\right)=0
$$


For $\theta_{i}=\pi$ and $M_{i}=\sigma_{i}$, additional linear equation for each non-oscillating transient roots $(p=1,2 . . n-2)$ can be written as,

$$
\sum_{i=0}^{n} \alpha_{i}\left(\sigma_{p}\right)^{\sigma_{i}} \cos \left(\pi \sigma_{i}\right)=0
$$

Equations (23) and (24) are reduced to equations (13) and (19) for integer values of derivative order $\sigma_{i}$. The remarks given in previous section are also valid for fractional order system.

Design procedure for oscillating high-order LTI system $(n>3)$ with no transient oscillation can be summarized as follows:

Step 1: Set $\alpha_{0}=1$ or any real number.

Step 2: Write $n$ number of linear equations such that equation (23) is for oscillating roots and equation (24) is for each nonoscillation roots.

Step 3: Solve linear equations to obtain $\alpha_{1}, \alpha_{2} . ., \alpha_{n}$ polynomials coefficients.

\section{NUMERICAL EXAMPLE}

In this section, we present an example system for fourth-order LTI systems, expressed in the form of,

$$
\alpha_{4} y^{(4)}+\alpha_{3} y^{(3)}+\alpha_{2} y^{(2)}+\alpha_{1} y^{\prime}+\alpha_{0} y=u
$$

In order to obtain harmonic oscillation at $\omega_{k}=2 \mathrm{radian} / \mathrm{sec}$ according to property 2 , one writes equation (13) for $n=4$ and then solves two equations given bellow,

$$
\alpha_{0}-4 \alpha_{2}+16 \alpha_{4}=0 \text { and } 2 \alpha_{1}-8 \alpha_{3}=0
$$

There are 5 design coefficients $\left(\alpha_{0}, \alpha_{1}, \alpha_{2}, \alpha_{3}\right.$ and $\left.\alpha_{4}\right)$ and two linear equations, so we choose three coefficients arbitrarily as $\alpha_{0}=1, \alpha_{1}=0.5$ and $\alpha_{4}=1$. Then, by solving equation (26), one obtains $\alpha_{2}=4.25$ and $\alpha_{3}=0.125$. Accordingly, the transfer function of oscillator can be obtained as,

$$
T(s)=\frac{1}{s^{4}+0.125 s^{3}+4.25 s^{2}+0.5 s+1}
$$

State space model in controller canonical form can be written as

$$
\begin{aligned}
& A=\left[\begin{array}{cccc}
-0.1250 & -4.25 & -0.5 & -1.0 \\
1.0 & 0 & 0 & 0 \\
0 & 1.0 & 0 & 0 \\
0 & 0 & 1.0 & 0
\end{array}\right], B=\left[\begin{array}{l}
1 \\
0 \\
0 \\
0
\end{array}\right], C^{T}=\left[\begin{array}{l}
0 \\
0 \\
0 \\
1
\end{array}\right], \\
& D=0 \quad(28)
\end{aligned}
$$

A Dirac function in the form of $u(t)=g \delta(t)$ was used for the input disturbance of the system. This imposes the initial condition of $y(0)=g$ and $y^{(i)}(0)=0, \quad i=1,2,3 . . n$. The amplitude of Dirac $(g)$ can be used for adjusting the amplitude of harmonic oscillation.
Figure 4(a) shows roots $\left(\lambda_{i}, i=0,1,2 . .4\right)$ in complex plane. Complex conjugate roots $\lambda_{1,2}= \pm 2 j$ yields harmonic oscillation at $\omega_{k}=2$ radian $/ \mathrm{sec}$ and the roots $\lambda_{3,4}=-0.0625 \pm 0.4961 j$ yield a transient decaying oscillation with a time constant $\tau=-1 /(-0.0625)=16 \mathrm{sec}$ at the angular frequency of $0.4961 \mathrm{radian} / \mathrm{sec}$. The transient oscillation almost attenuated in $5 \tau \cong 80 \mathrm{sec}$ and the harmonic oscillation at $\omega_{k}=2 \mathrm{radian} / \mathrm{sec}$ continues with an amplitude of 0.13 for $u(t)=\delta(t)$. In order to increase the amplitude of harmonic oscillation up to the amplitude of 0.65 as in Figure $4(\mathrm{c}), \quad u(t)=5 \delta(t)$ was applied from the input. This figure confirms that harmonic oscillation amplitude for the highorder LTI system can be controlled via the input signal $u(t)$.
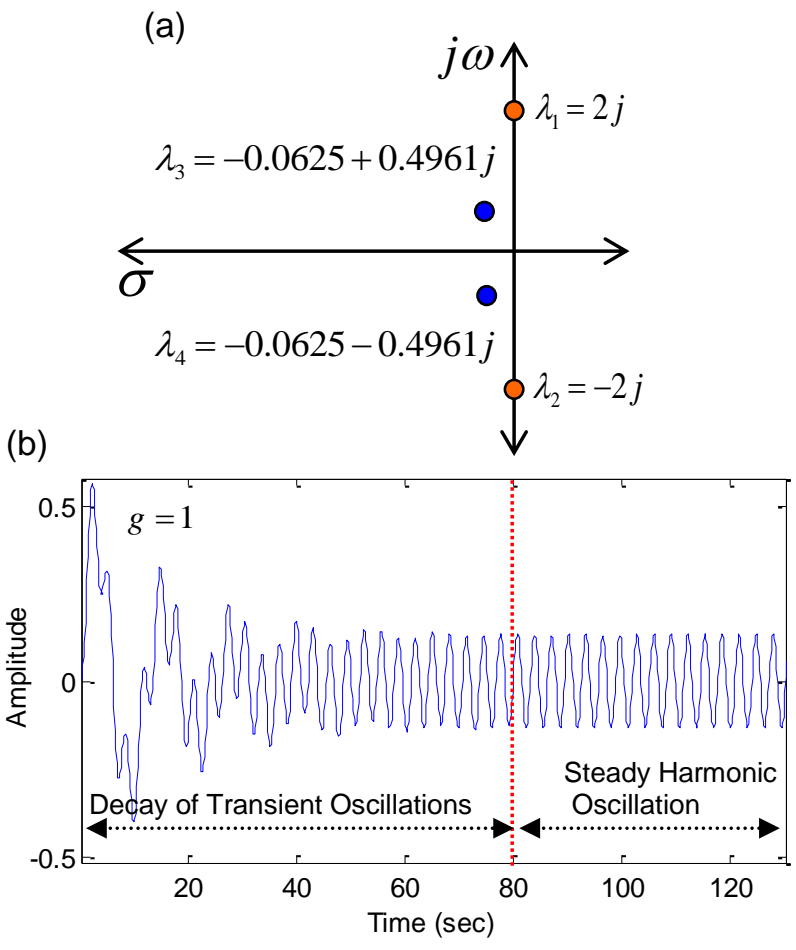

(c)

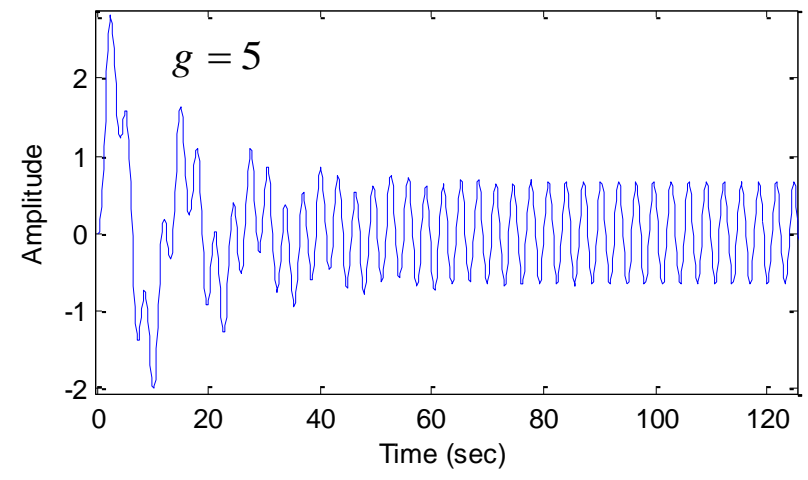

Fig. 4. (a) Root locations in complex plane, (b) LTI system response for $u(t)=\delta(t)$, (c) LTI system response for $u(t)=5 \delta(t)$ 
(a)

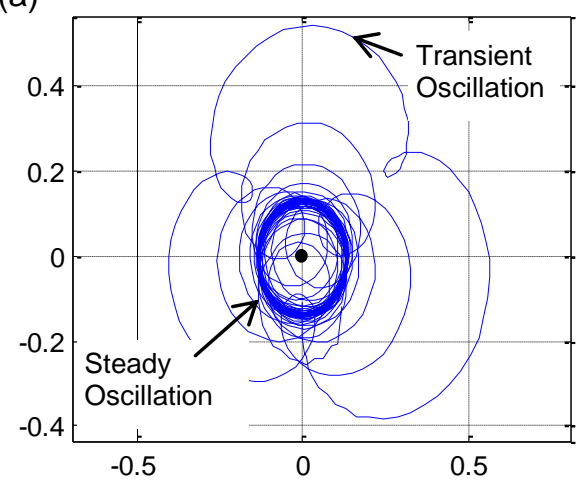

(b)

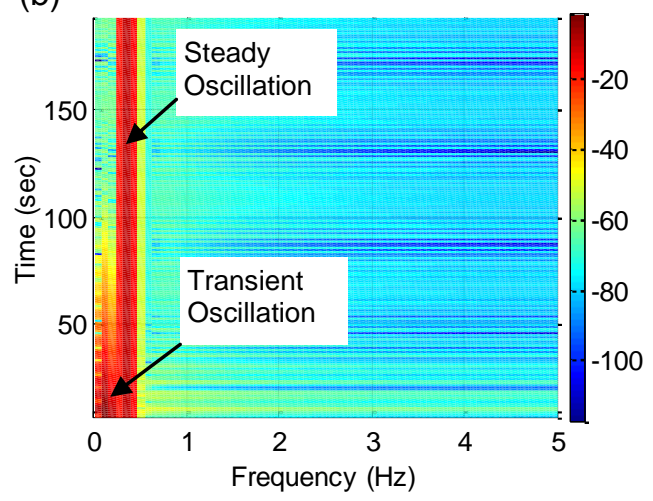

Fig. 5. (a) Discrete-time analytic representation of the output signal. (b) Spectrogram of the output signal

Figure 5(a) shows discrete-time analytic signal of the system output calculated by Hilbert transform. Analytic signal represents signals in a complex plane $\left(y(t)=|y(t)| e^{j<\phi(t)}\right)$, where vertical and horizontal axis are complex part and real part of complex signals. Irregular large circles were formed by transient oscillations in the figure. The circular orbit bands were formed by steady harmonic oscillation obtained at $\omega_{k}=2 \mathrm{radian} / \mathrm{sec}$. This effect can be clearly observed in Figure 5(b) demonstrating spectrogram of the system output. Spectrogram is obtained by moving window short-time Fourier transform of signals. This figure shows damping of transient oscillation at roughly $0.08 \mathrm{~Hz}$ (angular frequency of $0.4961 \mathrm{radian} / \mathrm{sec}$ ) in about $80 \mathrm{sec}$ and a steady harmonic oscillation at $0.31 \mathrm{~Hz}$ (angular frequency of $2 \mathrm{radian} / \mathrm{sec}$ ).
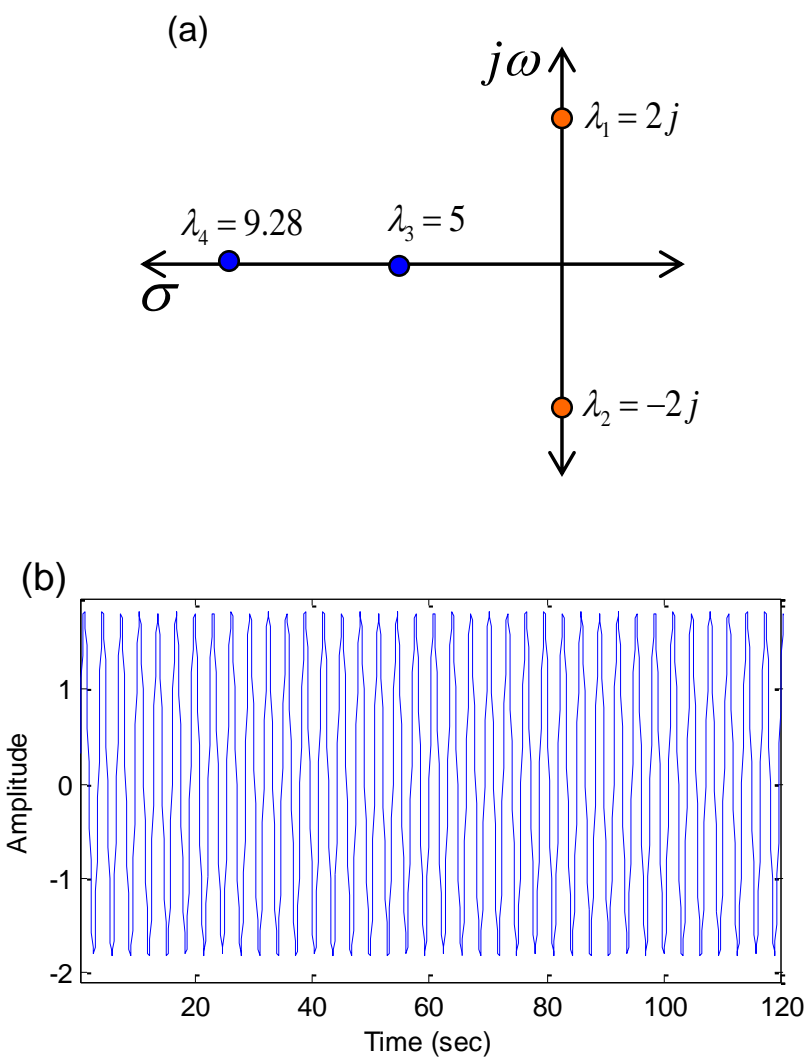

Fig. 6. (a) Root locations in complex plane, (b) LTI system response for $u(t)=\delta(t)$,

In order to remove transient oscillations caused from the complex conjugates roots $\lambda_{3,4}=-0.0625 \pm 0.4961 j$, we applied the design procedure for oscillating high-order LTI system with no transient oscillation given in previous section. For this proposes, we preferred decay of non-oscillating two transient components for $\sigma_{3}=5$ and $\sigma_{4}=9.2$ as in illustrated Figure 6(a). Two addition linear equations were written according to equation (19) as,

$$
\begin{array}{r}
\alpha_{0}-5 \alpha_{1}+25 \alpha_{2}-125 \alpha_{3}+625 \alpha_{4}=0 \& \\
\alpha_{0}-10 \alpha_{1}+100 \alpha_{2}-1000 \alpha_{3}+10000 \alpha_{4}=0
\end{array}
$$

For $\alpha_{0}=1$, the solutions of equations (26) and (29) were obtained as $\quad \alpha_{1}=0.3077, \alpha_{2}=0.2715, \quad \alpha_{3}=0.0769$, $\alpha_{4}=0.0054$ and the differential model of the system can be written as

$$
0.0054 y^{(4)}+0.0769 y^{(3)}+0.2715 y^{(2)}+0.3077 y^{\prime}+y=u
$$


(a)

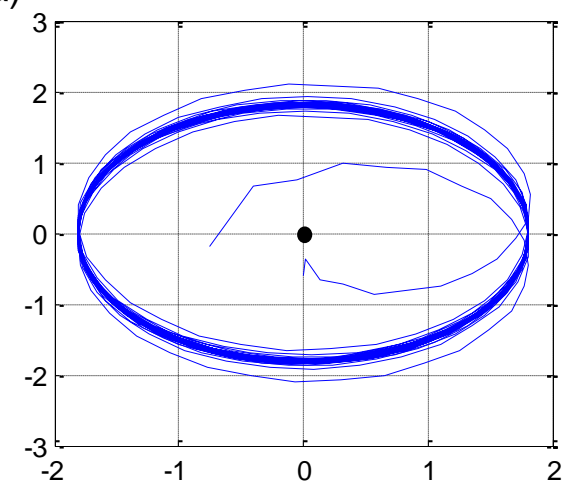

(b)

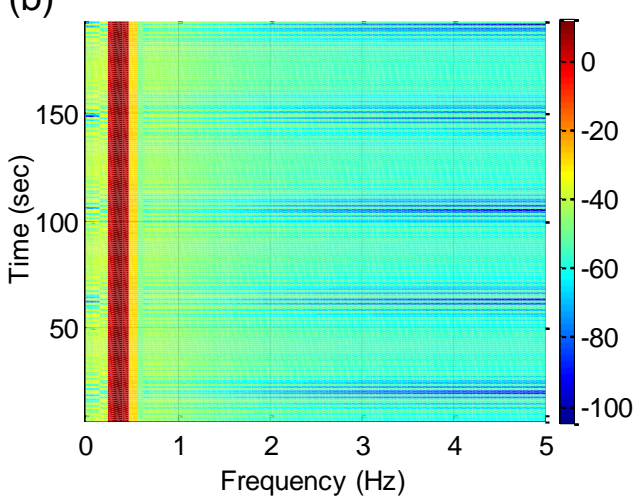

Fig. 7. (a) Discrete-time analytic representation of the output signal. (b) Spectrogram of the output signal

Figure 6(b) shows the system output, where transient oscillations are cleared. To better evaluate harmonic oscillations, Figures 7(a) and 7(b) shows discrete-time analytic signal and spectrogram of the system output. It is evident from figures that transient oscillations were cleared from the system output.

One of the noteworthy property of this oscillator is that one can add a bias level for harmonic oscillation by applying a step function $(u(t)=1, t \geq 0)$ to the input. Figure 8 (a) and (b) shows step response and discrete-time analytic signal of the system. A bias with a magnitude of one is apparent in these figures. Figure 9 shows results of variable step input applied for a fourth-order LTI system designed for $\omega_{k}=1 \mathrm{radian} / \mathrm{sec}$, $\sigma_{3}=5$ and $\sigma_{4}=9.8$. The differential model of the LTI oscillation control system was obtained as

$$
0.0204 y^{(4)}+0.3020 y^{(3)}+1.0204 y^{(2)}+0.3020 y^{\prime}+y=u(t)
$$

To better demonstrate amplitude and bias control of oscillations at $\omega_{k}=1 \mathrm{radian} / \mathrm{sec}$ via input signal $u(t)$, we applied subsequent delayed Dirac input and a variable step input as control signal. The subsequent delayed Dirac signal was formed as follows,

$$
u(t)=0.5 \delta(t)+\delta(t-100)+1.2 \delta(t-150)
$$

And, the following variable step input signal was used to disturb the oscillating system.

$$
u(t)=\left\{\begin{array}{cc}
0.5 & , t \leq 50 \\
1 & , 50<t \leq 100 \\
2 & , 100<t \leq 200
\end{array}\right.
$$
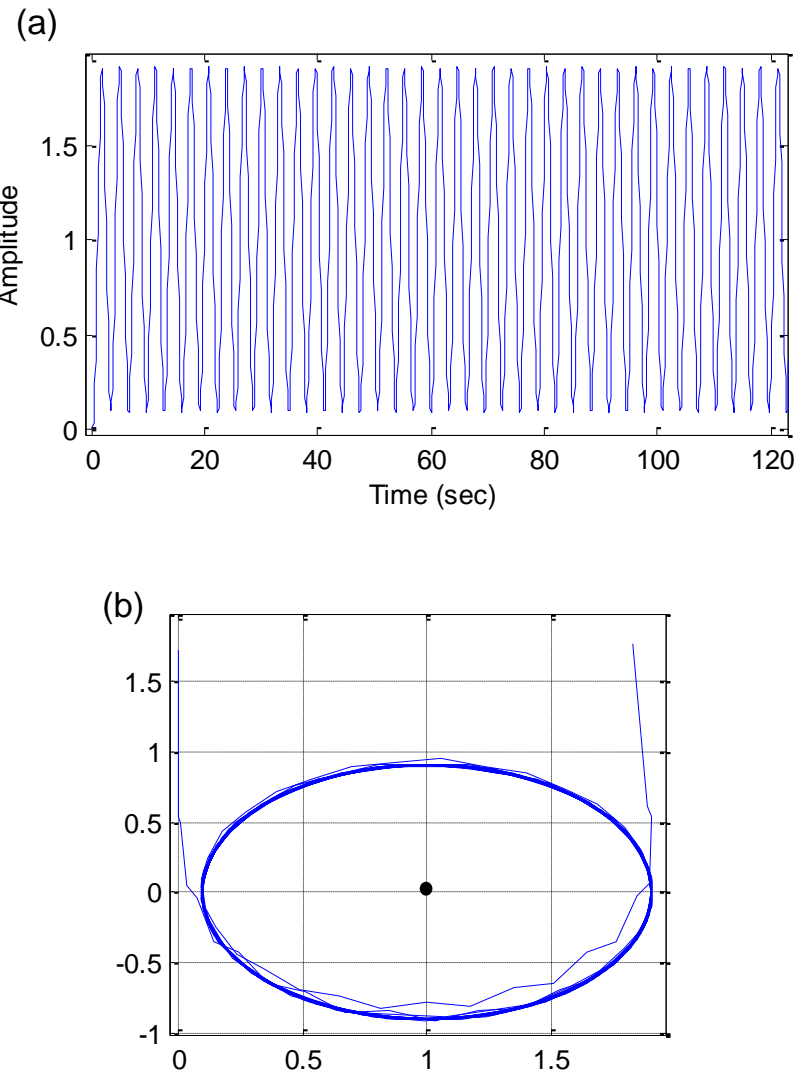

Fig. 8. (a) LTI system response for $u(t)=1$ exhibiting a bias level of one, (b) Discrete-time analytic representation of the output signal with a bias level of one.

Figure 9(a) and (b) demonstrates control of oscillation amplitude by the subsequent delayed Dirac signal generated by equation (32). Each pulse increases amplitude of harmonic oscillation for a zero bias. Figure 9(c) demonstrates shift of bias of oscillation by altering amplitude of the variable step function according to equation (33). It should be noticed that oscillation control is performed for amplitude by Dirac input and for bias by step input.

Practical harmonic oscillator implementation in circuit design encounters with voltage stability problems and it requires a control circuitry to sustain oscillation voltage stability[26]. 


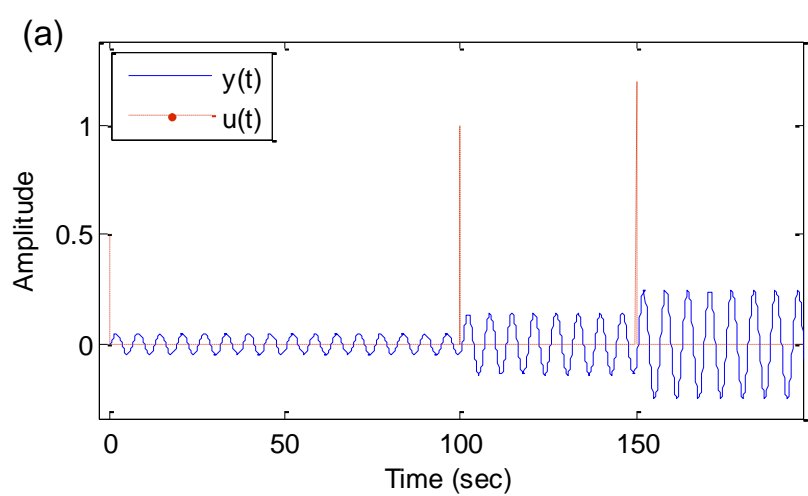

(b)
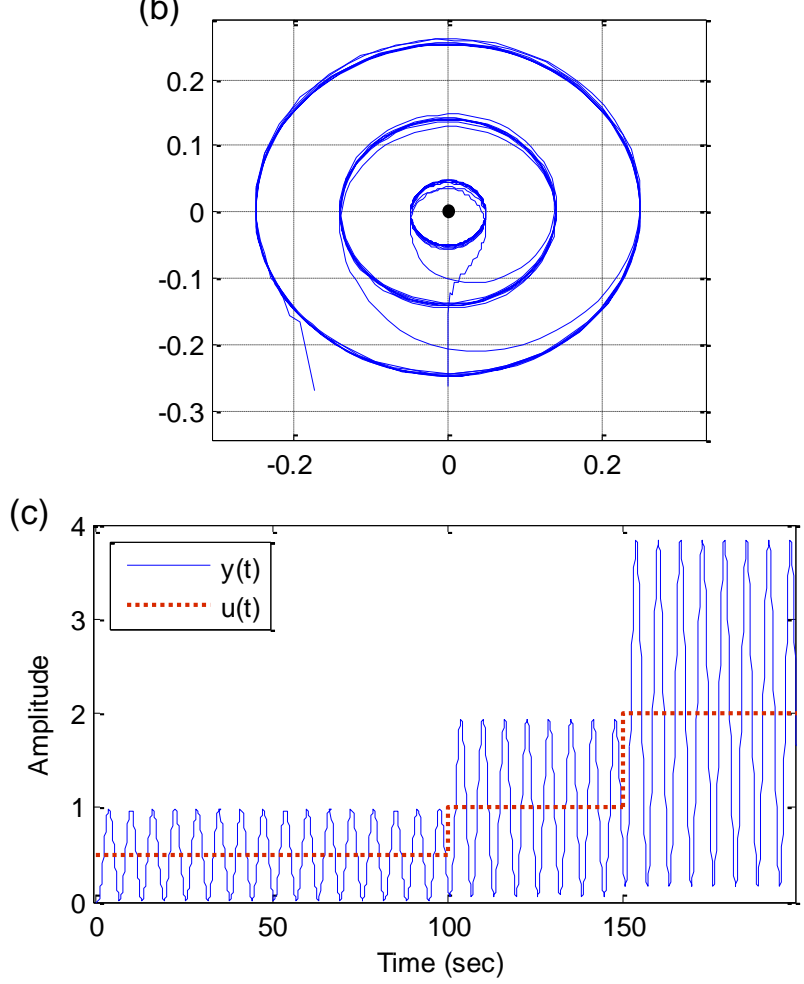

Fig. 9. (a) LTI system response for subsequent delayed dirac input, (b) Discrete-time analytic representation of the output signal for subsequent delayed dirac input, (c) Harmonic oscillation controlled with a variable step input and corresponding bias levels.

We present another example system for fractional-order LTI systems, expressed in the form of,

$$
\alpha_{3} y^{(3.6)}+\alpha_{2} y^{(2.4)}+\alpha_{1} y^{(1.2)}+\alpha_{0} y=u
$$

In order to obtain harmonic oscillation at $\omega_{k}=4$ radian/sec according to property 2 , one writes equation (23) for $n=3$ and then solves two equations given bellow,

$$
\begin{aligned}
& \alpha_{0}+0.8155 \alpha_{1}-1.8195 \alpha_{2}+1.6350 \alpha_{3}=0 \text { and } \\
& 2.5099 \alpha_{1}-1.3219 \alpha_{2}-1.1879 \alpha_{3}=0
\end{aligned}
$$

There are 4 design coefficients $\left(\alpha_{0}, \alpha_{1}, \alpha_{2}, \alpha_{3}\right)$ and two linear equations, $\alpha_{0}=1$ and $\alpha_{1}=0.5$ are chosen arbitrarily. Then, by solving equations (35), one obtains $\alpha_{2}=0.080733$ and $\alpha_{3}=0.013745$. Accordingly, the transfer function of fractional order LTI harmonic oscillator can be obtained as,

$$
T(s)=\frac{1}{0.01374 s^{3.6}+0.080733 s^{2.4}+0.5 s^{1.2}+1}
$$

A differential model of fractional order LTI harmonic oscillator was obtained as,

$$
0.013745 y^{(3.6)}+0.080733 y^{(2.4)}+0.5 y^{(1.2)}+y=u(t)
$$

Fig. 10 shows step response of fractional order LTI system and its discrete-time analytic representation. In order to simulate step response of equation (36), we used Matlab functions presented in [27]. We observed in simulation that amplitude of harmonic oscillation is not steady for a long time as harmonic oscillation of integer order LTI systems. One reason for this amplitude instability can be approximation errors, which are caused from approximate implementation of fractional order terms in numerical simulations.
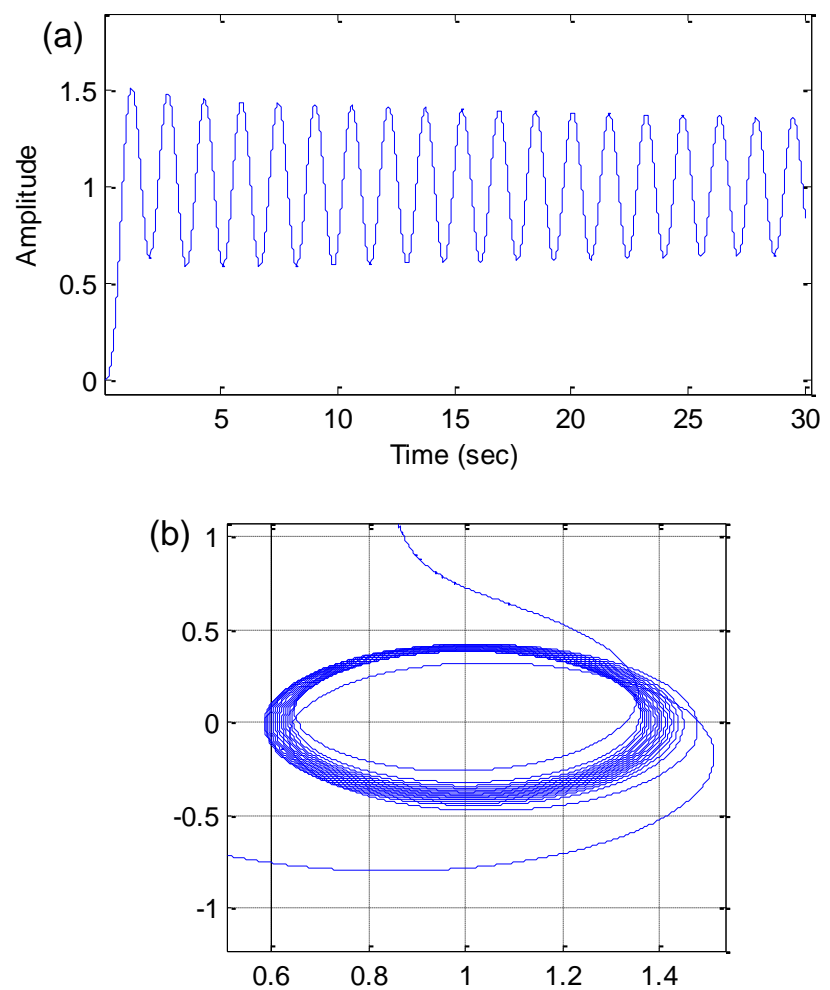

Fig. 10. (a) Step response of fractional order LTI system given by equation (36), (b) Discrete-time analytic representation of the output signal

\section{CONCLUSIONS}

This study presents a harmonic oscillation design and control procedure for high-order LTI systems. The paper discusses sufficient conditions of system coefficients to maintain 
harmonic oscillation and to clear transient harmonics from the [14] system output. Moreover, some properties related to oscillating LTI system solutions such as existence of solution, abundance of solutions, balanced solution families are discussed. Equation set for oscillating roots (equation (13)) and non-oscillation roots (equation (19)) placement is derived and a design procedure for oscillating high-order LTI systems with no transient oscillation is presented.

Design and control examples were presented for the fourthorder LTI systems. In these examples, we illustrated removal of undesired transient harmonic oscillation from the system response and controlling the amplitude and bias levels of harmonic oscillations of the systems via input signal.

In physics, harmonic oscillations are widely used to explain mechanisms acting on the periodic events. A notable point should be emphasized that the steady state behavior of a highorder LTI system can mimic a second-order harmonic oscillator, when the complex conjugate roots resulting in transient oscillations moves away the complex axis. This transient components damp down during the transient regime. Steady-state response analysis of physical systems can hide effects of higher-order eigenvalues or roots at the system output. However, the actual orders of harmonically oscillating systems can be estimated by evaluating transient response of systems.

Discussions and examples in the paper can be useful for system engineering and results can contribute to the field of vibration control, electrical systems, modeling and analysis of periodical physical phenomena.

\section{REFERENCES}

[1] Blackman P.F., The pole-zero approach to systems analysis, Rowse Muir Publications, 1962.

[2] Perkins W.R., Cruz J.B., Engineering of dynamic systems, Wiley, 1969.

[3] Harris C.J., Miles J.E., "Stability of linear systems : some aspects of kinematic similarity", Mathematics in Science and Engineering, Academic Press, 1980.

[4] Gopal M, Modern Control System Theory, New Age International, 1993, pp. 322-324.

[5] Dorf R.C. and Bishop R. H., Modern Control Systems. Addison-Wesley, New York, 1990.

[6] Tan N., "Computation of Stabilizing PI and PID controllers for processes with time delay", ISA Transactions, 44, 2005,pp.213-223.

[7] Moghaddam T.V. and Abbasi Y., "Tuning a Fractional Order PD and PID Controller with Lead Compensator for Integrating Time Delay Systems", Journal of Electrical and Control Engineering 2, 2012, pp.34-41.

[8] Wells J.D., "Effective Theories in Physics", Springer Briefs in Physics, 2012, pp.7-13.

[9] Chiorescu I., Bertet P., Semba1 K., Nakamura Y., Harmans C. J. P. M. and J. E. Mooij, "Coherent dynamics of a flux qubit coupled to a harmonic oscillator", Nature 431, 2004, pp.159-162.

[10] Carloni S.,Troisi A., Dunsby P. K. S., "Some remarks on the dynamical systems approach to fourth order gravity", General Relativity and Gravitation 41, 2009, pp.1757-1776.

[11] Yakovenko S., "Oscillation of Linear Ordinary Differential Equations: On a Theorem of A. Grigoriev", Journal of Dynamical and Control Systems, 12, 2006, pp.433-449.

[12] Ibrahim H.H., Tawfik M., "Limit-cycle Oscillations of Functionally Graded Material Plates Subject to Aerodynamic and Thermal Loads", Journal of Vibration and Control 16, 2010, pp.2147-2166.

[13] Kasnakoglu C., "Control of Oscillations in Flow Problems Under Frequency Limitations", Journal of Vibration and Control, 16, 2010, pp.1941-1966.
Saha A., Pandey S.S., Bhattacharya B., Wahi P., "Analysis and control of friction-induced oscillations in a continuous system", Journal of Vibration and Control 18, 2012, pp.467-480.

[15] Chen Y.M., Liu J.K., "Homotopy Analysis Method for Limit Cycle Oscillations of an Airfoil with Cubic Nonlinearities", Journal of Vibration and Control 16, 2009, pp.163-179.

[16] Brigante $M$ and Sumbatyan M.A., "An efficient method in 2D problem on transient oscillations of the elastic half-space interacting with a rigid structure", Journal of Vibration and Control, 2013, doi: $10.1177 / 1077546313490777$.

[17] Radwan A.G., Elwakil A.S., and Soliman A.M., "Fractional-Order Sinusoidal Oscillators: Design Procedure and Practical Examples", IEEE Transaction On Circuits And System 55, 2008, pp.2051-2063.

[18] Atay F.M., "Oscillation Control in Delayed Feedback Systems", Lecture Notes in Control and Information Sciences 273, 2002, pp.103116.

[19] Wilkins A.K., Tidor B., White J., Barton P.I., "Sensitivity Analysis for Oscillating Dynamic Systems", Siam J. Sci. Comput., 31, 2009, pp.2706-2732.

[20] Jolma I.W., Ni X.Y., Rensing L., Ruoff P., "Harmonic Oscillations in Homeostatic Controllers: Dynamics of the p53 Regulatory System", Biophysical Journal 98, 2010, pp.743-752.

[21] Kaplan, I. Gabay, G. Sarafian, D. Sarafian, "Biological applications of the "Filtered" Van der Pol oscillator", Journal of the Franklin Institute, 345, 2008,226-232.

[22] Abdelaziz T.H.S.,"Robust pole assignment for linear time-invariant systems using state-derivative feedback", Proceedings of the Institution of Mechanical Engineers, Part I: Journal of Systems and Control Engineering, 223, 2009, pp.187-199.

[23] Özgören M.K., "An algebraic method for designing controllers for multi-input multi-output linear systems via s-domain input-output decoupling", Proceedings of the Institution of Mechanical Engineers, Part I: Journal of Systems and Control Engineering, 223, 2009, 263274.

[24] Gross B. and Braga E.P., Singularities of Linear System Functions, Elsevier Publishing, New York, 1961.

[25] Bayat F.M. and Ghartemani M.K., "On the essential instabilities caused by multi-valued transfer functions", Math. Problems in Engineering Article ID 419046, 2008, 13p.

[26] Kaveh P., Ashrafi A. and Shtessel Y.B., "Robust Sliding Mode Harmonic Oscillator Suitable for Low Frequencies", System Theory, SSST '05 Proceedings of the Thirty-Seventh Southeastern Symposium, 2005, pp.249-252.

[27] Xue D., Chen Y.Q., Atherton D.P., "Linear Feedback Control Analysis and Design with MATLAB", Society for Industrial and Applied Mathematics, Philadelphia, 2007.

\section{BIOGRAPHIES}

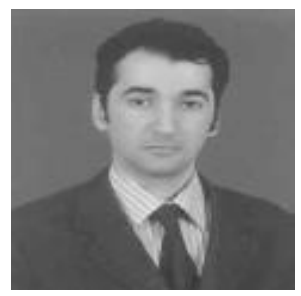

BARIS BAYKANT ALAGOZ was graduated from University of Istanbul Technical University department of Electronics and Communication Engineering in 1998. He worked for Alcatel Microelectronics and Turkish Telecom for several years. $\mathrm{He}$ is following $\mathrm{PhD}$ at Inonu University department of Department of Electrical-Electronics Engineering.

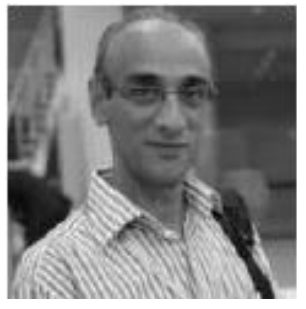

HAFIZ Z. ALİSOY was graduated from Moscow Technical University department of ElectroPhysics Engineering 1982. He had his PhD degree from USSR Science Academy Physics Institute of P.N. Lebedyev and Doctor of Sciences degree (DSc) from International Ecology-Energy Academy. He became as Full Professor in 1995. He received award of Young Scientist. He works at Namık Kemal University, Department of Electronics and Telecommunication Engineering. 